\title{
A NOTE ON $*(x, y, z)$-SIMPLE RINGS
}

\author{
MARTA NOWAKOWSKA \\ Institute of Mathematics, University of Silesia in Katowice \\ Katowice, Poland \\ ORCID: 0000-0002-6160-1411_E-mail: marta.nowakowska@us.edu.pl
}

\begin{abstract}
The main goal of this paper is to prove a correct version of one of the main results in the paper Note on some ideals of associative rings by M. Filipowicz, M. Kępczyk [Acta Math. Hungar. 142 (2014), 72-79]. Moreover, we give a new proof of Theorem 8 there.
\end{abstract}

1. Introduction and preliminaries. All rings in this paper are associative but not necessarily with unity.

We write $I \triangleleft_{t} R\left(I \triangleleft_{l} R, I \triangleleft_{r} R\right)$, if $I$ is a two-sided ideal (left ideal, right ideal) of a $\operatorname{ring} R$.

For a given ring $R$, we denote by $R^{1}$ the ring obtained by adjoining a unity to $R$ and by $R^{\text {op }}$ the ring with the opposite multiplication.

In 3] E. R. Puczyłowski introduced the notion of $*$-ideals, which is related to radical theory of rings. He defined $*$-simple rings (i.e. rings without non-trivial $*$-ideals) which are important examples of unequivocal rings. Later, in [2], the authors considered left *-ideals. Finally, in [1] a generalization of $*$-ideals and left $*$-ideals was introduced, i.e. the notion of $*(x, y, z)$-ideals, where $x, y, z \in\{l, r, t\}$.

Definition 1.1 ([1, Definition 1]). Let $x, y, z \in\{l, r, t\}$. A subring $I$ of a ring $R$ such that $I \triangleleft_{x} R$ is called a $*(x, y, z)$-ideal of $R$, if $I \triangleleft_{z} A$ for every $\operatorname{ring} A$ such that $R \triangleleft_{y} A$.

In our notation, $*$-ideals and left $*$-ideals are $*(t, t, t)$-ideals and $*(l, l, l)$-ideals, respectively.

A ring containing no non-trivial $*(x, y, z)$-ideals is called a $*(x, y, z)$-simple ring. The class of $*(x, y, z)$-simple rings will be denoted by $\mathcal{S}(x, y, z)$.

2010 Mathematics Subject Classification: 16D25.

Key words and phrases: $*(x, y, z)$-ideal, $*(x, y, z)$-simple ring.

The paper is in final form and no version of it will be published elsewhere. 
In the paper we prove that there exist $*(x, y, z)$-simple rings with zero multiplication which are not algebras over a field. This shows that Lemma 7 in [1, needed in the proof of Theorem 8 in 1], is not true. Next, we present a new, correct proof of Theorem 8 without using Lemma 7.

2. Results. We begin this section with an easy to observe property of $*(x, y, z)$-simple rings.

Lemma 2.1. Let $x, y \in\{l, r, t\}$. Then $R \in \mathcal{S}(x, y, r)$ if and only if $R^{\mathrm{op}} \in \mathcal{S}\left(x^{\prime}, y^{\prime}, l\right)$, where $l^{\prime}=r, r^{\prime}=l, t^{\prime}=t$.

Obviously, there are 27 classes $\mathcal{S}(x, y, z)$, where $x, y, z \in\{l, r, t\}$. The next three facts show that there are 12 classes among them which consist of all associative rings.

Proposition 2.2. Let $x \in\{l, r, t\}$. Then the class $\mathcal{S}(x, r, l)$ is equal to the class of all rings.

Proof. Assume $R$ is an associative ring and $I$ is a $*(x, r, l)$-ideal of $R$, where $x \in\{l, r, t\}$. Note that $R \cong\left(\begin{array}{cc}R & 0 \\ 0 & 0\end{array}\right)<_{r}\left(\begin{array}{cc}R^{1} & 0 \\ R^{1} & 0\end{array}\right)$. Since $I \cong\left(\begin{array}{cc}I & 0 \\ 0 & 0\end{array}\right)$ is a $*(x, r, l)$-ideal of $R$, $\left(\begin{array}{ll}I & 0 \\ 0 & 0\end{array}\right)<_{l}\left(\begin{array}{cc}R^{1} & 0 \\ R^{1} & 0\end{array}\right)$. Then $\left(\begin{array}{ll}R^{1} & 0 \\ R^{1} & 0\end{array}\right)\left(\begin{array}{ll}I & 0 \\ 0 & 0\end{array}\right)=\left(\begin{array}{cc}R^{1} I & 0 \\ R^{1} I & 0\end{array}\right) \subseteq\left(\begin{array}{ll}I & 0 \\ 0 & 0\end{array}\right)$. The last inclusion implies the equality $R^{1} I=0$. Therefore $I=0$. This means that $R \in \mathcal{S}(x, r, l)$.

Below we present an immediate consequence of the above proposition and Lemma 2.1 .

Corollary 2.3. Let $x \in\{l, r, t\}$. Then the class $\mathcal{S}(x, l, r)$ is equal to the class of all rings.

Note that $*(x, r, t)$-ideals are $*(x, r, l)$-ideals and $*(x, l, t)$-ideals are $*(x, l, r)$-ideals, for every $x \in\{l, r, t\}$. Therefore the inclusions $\mathcal{S}(x, r, l) \subseteq \mathcal{S}(x, r, t)$ and $\mathcal{S}(x, l, r) \subseteq \mathcal{S}(x, l, t)$ hold. Thus by Proposition 2.2 and Corollary 2.3 we obtain the following result.

Corollary 2.4. Let $x \in\{l, r, t\}$. Then:

(i) The class $\mathcal{S}(x, r, t)$ is equal to the class of all rings.

(ii) The class $\mathcal{S}(x, l, t)$ is equal to the class of all rings.

Remark 2.5. Proposition 2.2 and Corollaries 2.3 and 2.4 show that Lemma 7 in [1] saying that a $*(x, y, z)$-simple ring with zero multiplication is an algebra over a field, for every $x, y, z \in\{l, r, t\}$, is not true.

Now we are ready to present a corrected version of Lemma 7 . The proof remains the same as that in [1. We will denote by $\mathcal{A}$ any class of rings appearing in Proposition 2.2 and Corollary 2.3 .

Corollary 2.6. Let $x, y, z \in\{l, r, t\}, R \in \mathcal{S}(x, y, z)$ and $R \notin \mathcal{A}$. If $R$ is a ring with zero multiplication, then $R$ is an algebra over a field.

The next two results allow us to prove Theorem 8 in [1] without using Lemma 7. 
LEMMA 2.7. The following equalities hold:

(i) $\mathcal{S}(l, t, t)=\mathcal{S}(t, t, t)$.

(ii) $\mathcal{S}(r, t, l)=\mathcal{S}(t, t, l)$.

(iii) $\mathcal{S}(r, t, t)=\mathcal{S}(t, t, t)$.

(iv) $\mathcal{S}(l, t, r)=\mathcal{S}(t, t, r)$.

Proof. (i): Two-sided ideals are left ideals, hence $*(t, t, t)$-ideals are $*(l, t, t)$-ideals. This proves the inclusion $\mathcal{S}(l, t, t) \subseteq \mathcal{S}(t, t, t)$.

To show the reverse inclusion, let $R \in \mathcal{S}(t, t, t)$ and $J$ be a $*(l, t, t)$-ideal of $R$. Obviously $J<_{l} R \triangleleft R$, hence $J \triangleleft R$. This means that $J$ is a $*(t, t, t)$-ideal of $R \in \mathcal{S}(t, t, t)$. Consequently, either $J=0$ or $J=R$, so $R \in \mathcal{S}(l, t, t)$.

(ii): Note that $*(t, t ; l)$-ideals are $*(r, t ; l)$-ideals. Therefore the inclusion $\mathcal{S}(r, t ; l) \subseteq$ $\mathcal{S}(t, t ; l)$ is clear.

Assume $R \in \mathcal{S}(t, t, l)$ and $J$ is a $*(r, t, l)$-ideal of $R$. Then $J<_{r} R \triangleleft R$ and by the assumption we obtain $J<_{l} R$. Thus $J$ is a $*(t, t, l)$-ideal of $R \in \mathcal{S}(t, t, l)$. Hence, either $J=0$ or $J=R$. Finally, $\mathcal{S}(t, t, l) \subseteq \mathcal{S}(r, t, l)$.

Statements (iii) and (iv) directly follow from Lemma 2.1 and the above statements (i) and (ii), respectively.

LEMMA 2.8. The following equalities hold:

(i) $\mathcal{S}(t, t ; l)=\mathcal{S}(t, t ; t)$.

(ii) $\mathcal{S}(t, t, r)=\mathcal{S}(t, t, t)$.

Proof. (i): $*(t, t, t)$-ideals are $*(t, t, l)$-ideals, hence $\mathcal{S}(t, t, l) \subseteq \mathcal{S}(t, t, t)$.

To prove the opposite implication let $R \in \mathcal{S}(t, t, t)$. Then by Theorem 1 in [3] we know that $R$ is either a simple ring or an algebra with zero multiplication over a field. If $R$ is a simple ring, then obviously $R \in \mathcal{S}(t, t, l)$. If $R$ is an algebra with zero multiplication over a field, then applying Lemma 6 in [1], we get again that $R \in \mathcal{S}(t, t, l)$.

Applying Lemma 2.1 and the above statement (i) we obtain (ii).

Using the above results we are able to give a new proof of the following characterization from [1].

Theorem 2.9 ([1], Theorem 8]). Let $x, y \in\{l, r, t\}$. Then $R \in \mathcal{S}(x, t, y)$, where $x=y=t$ or $x \neq y$ if and only if $R$ is either a simple ring or an algebra with zero multiplication over a field.

Proof. Assume $x, y \in\{l, r, t\}$ and $x=y=t$ or $x \neq y$. Lemmas 2.7 and 2.8 imply the equality $\mathcal{S}(x, t, y)=\mathcal{S}(t, t, t)$. Now, the required equivalence follows from Theorem 1 in 3 .

\section{References}

[1] M. Filipowicz, M. Kępczyk, Note on some ideals of associative rings, Acta Math. Hungar. 142 (2014), 72-79. 
[2] M. Filipowicz, E. R. Puczyłowski, On left ideals of associative rings, Zeszyty Naukowe Politechniki Białostockiej 20 (2011), 15-21.

[3] E. R. Puczyłowski, On unequivocal rings, Acta Math. Acad. Sci. Hungar. 36 (1980), 57-62.

[4] A. D. Sands, On ideals in over-rings, Publ. Math. Debrecen 35 (1988), 273-279.

[5] S. Veldsman, Extensions and ideals of rings, Publ. Math. Debrecen 38 (1991), 297-309. 